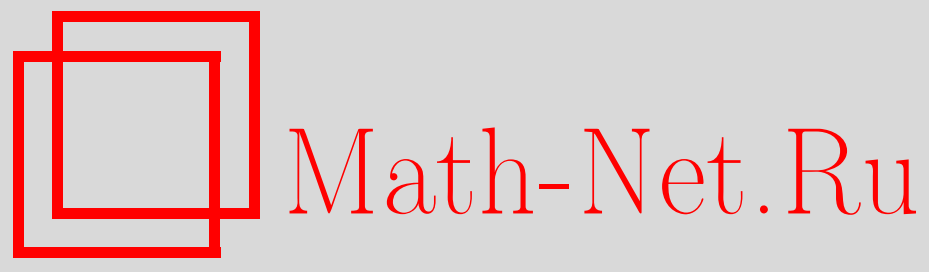

А. В. Лебедев, Двойной показательный закон для максимальных ветвящихся процессов, Дискрет. матем., 2002, том 14, выпуск 3, 143-148

DOI: https://doi.org/10.4213/dm261

Использование Общероссийского математического портала Math-Net.Ru подразумевает, что вы прочитали и согласны с пользовательским соглашением http: //www.mathnet.ru/rus/agreement

Параметры загрузки:

IP : 54.147 .182 .235

26 апреля 2023 г., 14:37:13

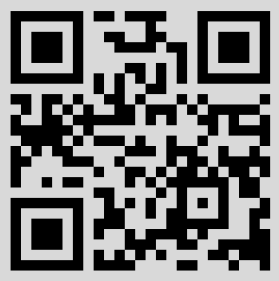


Удк 519.218

\title{
Двойной показательный закон для \\ максимальных ветвящихся процессов
}

\author{
๑) 2002 г. А. В. Лебедев
}

Рассматриваются максимальные ветвящиеся процессы, заданные рекуррентной формулой

$$
Z_{n+1}=\bigvee_{m=1}^{Z_{n}} \xi_{m, n},
$$

где через $\vee$ обозначена операция взятия максимума, $\xi_{m, n}, m \geqslant 1, n \geqslant 0$, независимы и имеют функцию распределения $F$ на $\mathbf{Z}_{+}$.

Доказаны предельные теоремы для стационарных распределений процессов $\left\{Z_{n}^{(N)}\right\}$ с $F^{(N)}(x)=F^{N}(x)$ при $N \rightarrow \infty$, когда $F$ принадлежит области притяжения двойного показательного закона.

Работа выполнена при поддержке Российского фонда фундаментальных исследований, проект 00-01-00131.

\section{1. Введение}

Классическими объектами исследования в теории случайных процессов являются ветвящиеся процессы Гальтона-Ватсона $[1,2]$, исследования различных аспектов и модификаций которых продолжаются [3, 4].

Дж. Ламперти [5, 6] ввел в рассмотрение максимальные ветвящиеся процессы, заданные рекуррентной формулой

$$
Z_{n+1}=\bigvee_{m=1}^{Z_{n}} \xi_{m, n},
$$

где через $\vee$ обозначена операция взятия максимума, и $\xi_{m, n}, m \geqslant 1, n \geqslant 0,-$ независимые случайные величины с общей функцией распределения $F$ на $\mathbf{Z}_{+}$. Полагаем (как и в случае суммирования), что ревультат взятия максимума ноль раз (при $Z_{n}=0$ ) равен нулю.

Можно сказать (по аналогии с процессами Гальтона-Ватсона), что в максимальном ветвящемся процессе (MBП) выживают потомки только одной частицы, у которой их больше всего. Понятно также, что множество возможных значений МВП (при $n \geqslant 1$ ) совпадает с множеством возможных значений числа потомков. Из (1) следует, что процесс является однородной цепью Маркова на этом множестве. 
В [5] показано, что если $F(0)=0$, то при выполнении условия

$$
\limsup _{x \rightarrow \infty} x(1-F(x))<e^{-\gamma},
$$

где $\gamma=0,577 \ldots$ - константа Эйлера, цепь $\left\{Z_{n}\right\}$ положительно возвратна, и напротив, при

$$
\liminf _{x \rightarrow \infty} x(1-F(x))>e^{-\gamma}
$$

имеет место сходимость $Z_{n} \rightarrow \infty$ при $n \rightarrow \infty$ почти наверное (П. н.).

Далее, в [6] рассмотрен критический случай, когда

$$
x(1-F(x)) \rightarrow e^{-\gamma}
$$

при $x \rightarrow \infty$, с учетом дальнейших членов разложения хвоста на бесконечности. Показано, что если $\left(e^{\gamma} x(1-F(x))-1\right) \ln x \rightarrow d$ при $x \rightarrow \infty$, то процесс возвратен при $d<\pi^{2} / 12$ и уходит на бесконечность п. н. при $d>\pi^{2} / 12$.

Далее, полагая везде $F(0)=0$, будем рассматривать МВП при более сильном, чем (2), условии конечности математического ожидания числа потомков

$$
a=\int_{0}^{\infty}(1-F(u)) d u<\infty,
$$

из которого следует, что

$$
\lim _{x \rightarrow \infty} x(1-F(x))=0 .
$$

Далее будем использовать обозначение $\bar{F}(x)=1-F(x)$.

Рассмотрим последовательность МВП $\left\{Z_{n}^{(N)}\right\}$ с $F^{(N)}(x)=F^{N}(x), N \geqslant 1$. Пусть $F$ удовлетворяет условию (4), тогда в силу асимптотики

$$
\bar{F}^{(N)}(x)=1-F^{(N)}(x) \sim N \bar{F}(x)
$$

при $x \rightarrow \infty$, для $F^{(N)}$ при любом фиксированном $N$ также выполнено соотношение

$$
\lim _{x \rightarrow \infty} x\left(1-F^{N}(x)\right)=0
$$

аналогичное (4). Из положительной возвратности $\left\{Z_{n}^{(N)}\right\}$ в сочетании с неприводимостью и апериодичностью следует эргодичность и существование стационарного предельного распределения $\Psi^{(N)}$ у данной цепи Маркова (при каждом фиксированном $N \geqslant 1$ ).

Случайные величины с распределениями $\Psi^{(N)}$ будем обозначать через $\tilde{Z}^{(N)}$. Из (1) получаем, что

$$
\tilde{Z}^{(N)} \stackrel{d}{=} \bigvee_{m=1}^{\tilde{Z}^{(N)}} \xi_{m}^{(N)}
$$

где $\xi_{m}^{(N)}, m \geqslant 1$, независимы и имеют функцию распределения $F^{(N)}$.

Основной целью работы является нахождение асимптотики для $\Psi^{(N)}$ при $N \rightarrow \infty$ в случае, когда $F$ принадлежит области притяжения двойного показательного закона

$$
\Lambda(x)=\exp \left\{-e^{-x}\right\}
$$




\section{2. Вспомогательные утверждения}

Определим функщию

$$
\varphi(s)=\int_{0}^{\infty}\left(1-F^{s}(x)\right) d x, \quad s>0 .
$$

При натуральных значениях аргумента

$$
\varphi(k)=\mathbf{M} \bigvee_{m=1}^{k} \xi_{m}, \quad k \geqslant 1,
$$

где $\xi_{m}, m \geqslant 1$, независимы и имеют функцию распределения $F$.

Лемма 1. Если верно (3), то $\varphi(s)$ конечна при всех $s \geqslant 0$.

Доказательство. Поскольку $1-F(x)^{s} \sim s \bar{F}(x)$ при $x \rightarrow \infty$, из сходимости интеграла (3) следует сходимость интеграла (6) при всех $s \geqslant 0$.

Лемма 2. Функция $\varphi$ не убывает и вогнута.

Утверждение следует из монотонности и выпуклости экспоненты.

\section{3. Предельная теорема}

Пусть $F$ принадлежит области притяжения двойного показательного закона

$$
\Lambda(x)=\exp \left\{-e^{-x}\right\}
$$

при линейной нормировке, то есть существуют числовые последовательности $a_{k}>0, b_{k}$, $k \geqslant 1$ такие, что для любого фиксированного $x \in \mathbf{R}$

$$
\lim _{k \rightarrow \infty} \mathbf{P}\left(\bigvee_{m=1}^{k} \xi_{m} \leqslant a_{k} x+b_{k}\right)=\Lambda(x) .
$$

Критерии выполнения (7) можно найти в [7]. В частности, хвост $F$ должен убывать быстрее любой степени. Заметим, что поскольку мы рассматриваем дискретные распределения, хвост функции распределения $F$ также должен убывать медленнее любой экспоненты, так как в противном случае не выполняется необходимое условие существования невырожденного предельного распределения максимумов (см. [8]): при $\boldsymbol{n} \rightarrow \infty$

$$
\bar{F}(x) / \bar{F}(x-0) \rightarrow 1
$$

для любого $c>1$.

Определим функщию

$$
u(s)=\inf \{x: s \bar{F}(x) \leqslant 1\}, \quad s>0 .
$$

По теореме 2.1.3 из [7] значения $u(k), k \geqslant 1$, можно выбрать в качестве $b_{k}$, что и будем предполагать в дальнейшем. Заметим, что $u(s)$ монотонно не убывает и стремится к бесконечности при $s \rightarrow \infty$. 
Лемма 3. Если верно (7), то $\varphi(s) \sim u(s)$ при $s \rightarrow \infty$, причем $\varphi$ и и-медленно меняючиеся функиии.

Доказательство. Согласно теореме 4.1.1 в монографии [7] (с точностью до обозначений) $a_{k} / b_{k} \rightarrow 0$ и при $k \rightarrow \infty$

$$
\max \left\{\xi_{1}, \ldots, \xi_{k}\right\} / b_{k} \stackrel{P}{\rightarrow} 1 .
$$

Как было отмечено еще в [8], для неограниченных сверху распределений этот закон больших чисел эквивалентен том, что при $x \rightarrow \infty$

$$
\bar{F}(c x) / \bar{F}(x) \rightarrow 0
$$

для любого $c>1$. По теореме 3.2 из [9] получаем, что $\varphi(k) \sim u(k)$ при $k \rightarrow \infty$.

Докажем теперь, что $u$ - медленно меняющаяся функция. Пусть существует числовая последовательность $s_{m}>0, m \geqslant 1$, а также числа $p, q>1$ такие, что $u\left(p s_{m}\right) \geqslant q u\left(s_{m}\right)$ для всех $m \geqslant 1$, и $s_{m} \rightarrow \infty$ при $m \rightarrow \infty$. Тогда

$$
\liminf _{m \rightarrow \infty} \frac{\bar{F}\left(q u\left(s_{m}\right)\right)}{\bar{F}\left(u\left(s_{m}\right)\right)} \geqslant \lim _{m \rightarrow \infty} \frac{\bar{F}\left(u\left(p s_{m}\right)\right)}{\bar{F}\left(u\left(s_{m}\right)\right)}=\frac{1}{p}>0,
$$

что противоречит (8). Делая замены $p^{\prime}=1 / p, q^{\prime}=1 / q, s_{m}^{\prime}=p s_{m}$ мы также доказываем, что не существует последовательности $s_{m}^{\prime}$ такой, что $u\left(p^{\prime} s_{m}^{\prime}\right) \leqslant q^{\prime} u\left(s_{m}^{\prime}\right)$ для всех $m \geqslant 1$.

Из того, что $u$ медленно меняется, следует, что $u(k+1) / u(k) \rightarrow 1$ при $k \rightarrow \infty$, откуда, в свою очередь, следует, что $\varphi(k+1) / \varphi(k) \rightarrow 1$ при $k \rightarrow \infty$. Следовательно, $\varphi(s) \sim u(s)$ при $s \rightarrow \infty$, и $\varphi$ также оказывается медленно меняющейся функщией.

Введем обозначение $\mu_{N}=M \tilde{Z}^{(N)}$. Из $F(0)=0$ следует, что $\xi_{m} \geqslant 1, \tilde{Z}^{(N)} \geqslant 1$ п. н., и заведомо справедливы соотношения $a \geqslant 1, \varphi(s) \geqslant 1$ при $s \geqslant 1$, а также $\mu_{N} \geqslant 1$ при $N \geqslant 1$.

Лемма 4. Для любого $\varepsilon>0$ справедливо неравенство $\mu_{N} \leqslant \varphi\left(N^{1+\varepsilon}\right)$ при всех достаточно больших $N$.

Доказательство. Из (5) следует, что $\mu_{N}=\mathbf{M} \varphi\left(N \tilde{Z}^{(N)}\right)$, откуда с помощью лемм 1 и 2 получаем оценку

$$
\mu_{N} \leqslant \varphi\left(N \mu_{N}\right)
$$

Пусть $0<\delta<\varepsilon /(1+\varepsilon)<1$. По лемме $3 \varphi(s)=o\left(s^{\delta}\right)$ при $s \rightarrow \infty$. Следовательно, существует $C$ такое, что $\varphi(s) \leqslant C s^{\delta}$ при $s \geqslant 1$. Из (9) получаем, что $\mu_{N} \leqslant\left(C N^{\delta}\right)^{1 /(1-\delta)}$ при $N \geqslant 1$, откуда $\mu_{N} \leqslant N^{\varepsilon}$ при всех достаточно больших $N$. Повторно применяя (9), получаем утверждение леммы 4.

Введем дополнительное условие: пусть для любого $\theta>1$ существует $\varepsilon>0$ такое, что при $s \rightarrow \infty$

$$
s u\left(s^{1+\varepsilon}\right) \bar{F}(\theta u(s)) \rightarrow 0 .
$$


Заметим, что (10) имеет смысл ограничения тяжести хвоста $F$. Условиям (7) и (10) удовлетворяют распределения Вейбулла, показательное, гамма, нормальное. Разницу можно увидеть на примере класса распределений с такими хвостами, что $\bar{F}(x) \sim \exp \left\{-(\ln x)^{\alpha}\right\}$ при $x \rightarrow \infty$; тогда (7) выполняется при $\alpha>1$, а (10) при $\alpha>2$.

Приходится отметить, что условие (10), прямо используемое далее в доказательстве теоремы, не выглядит оптимальным. Вопрос о его необходимости, уточнении или снятии является предметом дальнейших исследований автора.

Теорема 1. Если выполнены условия (7) $и$ (10), то при $N \rightarrow \infty$

$$
\tilde{Z}^{(N)} / u(N) \stackrel{P}{\rightarrow} 1
$$

Доказательство. Пусть $\theta>1, \varepsilon>0$, тогда из (5), лемм 3 и 4 получаем, что при всех достаточно больших $N$

$$
\mathbf{P}\left(\tilde{Z}^{(N)}>\theta u(N)\right) \leqslant \mu_{N} \bar{F}^{(N)}(\theta u(N)) \leqslant N \varphi\left(N^{1+\varepsilon}\right) \bar{F}(\theta u(N))
$$

и при $N \rightarrow \infty$

$$
N \varphi\left(N^{1+\varepsilon}\right) \bar{F}(\theta u(N)) \sim N u\left(N^{1+\varepsilon}\right) \bar{F}(\theta u(N)) \rightarrow 0 .
$$

Пусть теперь $\theta<1$, тогда в силу (8) при $N \rightarrow \infty$

$$
\mathbf{P}\left(\tilde{Z}^{(N)} \leqslant \theta u(N)\right) \leqslant F(\theta u(N))^{N} \rightarrow 0
$$

Следствие 1. Если выполнены условия (7) и (10), то для любого фиксированного $x \in \mathbf{R}$

$$
\lim _{N \rightarrow \infty} \mathbf{P}\left(\tilde{Z}^{(N)} \leqslant a_{k(N)} x+b_{k(N)}\right)=\Lambda(x)
$$

для любых $k(N) \sim N u(N)$ при $N \rightarrow \infty$.

Утверждение следует из (5), (11) и теоремы 6.2.1 в [7].

Пример 1. Пусть $F(n)=1-\exp \{-\sqrt{n}\}$, тогда $a_{k}=2 \ln k, b_{k}=(\ln k)^{2}$ при $k \geqslant 1$, и следовательно, при $N \rightarrow \infty$

$$
\mathbf{p}\left(\frac{\tilde{Z}^{(N)}-(\ln N+2 \ln \ln N)^{2}}{2(\ln N+2 \ln \ln N)} \leqslant x\right) \rightarrow \Lambda(x) .
$$

Автор благодарен рецензенту за полезные замечания и предложения. 


\section{Список литературы}

1. Севастьянов Б. А., Ветвячиеся прочессы. Наука, Москва, 1971.

2. Ватутин В. А., Зубков А. М., Ветвящиеся процессы. І. В кн.: Итоги науки и техники. Теория вероятн. Матем. статистика. Теор. кибернетика. ВИНИТИ, Москва, 1985. с. 3-67.

3. Колчин В. Ф., Ветвящиеся процессы и случайные гипердеревья. Дискретная математика (1999) 11, №1, 8-23.

4. Севастьянов Б. А., Минимальные точки надкритического ветвящегося случайного блуждания на решетке $\mathbf{N}_{0}^{r}$ и многотипные ветвящиеся процессы Гальтона-Ватсона. Дискретная математика (2000) 12, №1, 3-6.

5. Lamperti J., Maximal branching processes and long-range percolation. J. Appl. Probab. (1970) 7, №1, 89-96.

6. Lamperti J., Remarks on maximal branching processes. Теория вероятностей и ее применения (1972) 17, №1, 46-54.

7. Галамбош Я. И., Асимптотическая теория экстремальных порядковых статистик. Наука, Москва, 1984.

8. Gnedenko B. V., Sur la distribution limite du terme maximum d'une série aléatoire. Ann. Math. (1943) 44, №3, 423-453.

9. Pickands J., Moment convergence of sample extremes. Ann. Math. Stat. (1968) 39, №3, 881-889.

Статья поступила 13.09.2001. Переработанный вариант поступил 20.12.2001. 